\title{
Oscillator-phase coupling for different two-dimensional network connectivities
}

\author{
Ernst Niebur, ${ }^{*}$ Heinz G. Schuster, ${ }^{\dagger}$ Daniel M. Kammen, ${ }^{\ddagger}$ and Christof Koch \\ Computation and Neural Systems Program, California Institute of Technology 216-76, Pasadena, California 91125
}

(Received 7 June 1991)

\begin{abstract}
We investigate the dynamics of large arrays of coupled phase oscillators driven by random intrinsic frequencies under a variety of coupling schemes, by computing the time-dependent cross-correlation function numerically for a two-dimensional array consisting of $128 \times 128$ oscillators as well as analytically for a simpler model. Our analysis shows that for overall equal interaction strength, a sparse-coupling scheme in which each oscillator is coupled to a small, randomly selected subset of its neighbors leads to a more rapid and robust phase locking than nearest-neighbor coupling or locally dense connection schemes.
\end{abstract}

PACS number(s): 42.66. $-\mathrm{p}$, 87.22. $-\mathrm{q}, 05.20 .-\mathrm{y}$

\section{INTRODUCTION}

Networks of interacting oscillators provide a model for numerous physical processes ranging from the behavior of magnetic materials [1], mode-locking lasers [2], and atmospheric dynamics [3] to the activity of populations of neurons in central pattern generators in vertebrates [4] as well as in the mammalian olfactory and visual cortex $[5,6]$. Two groups $[7,8]$ have recently reported highly synchronized, stimulus-specific oscillations in various areas of the visual cortex of anesthetized as well as awake cats. Neurons up to $7 \mathrm{~mm}$ apart show phase-locked oscillations with a phase shift of less than $1 \mathrm{msec}$ that have been proposed to play a role in the coding of visual information [9-14].

Many physical systems including the cortex are two dimensional (2D), at least to some approximation. The complexity of networks of even relatively simple units let alone "real" cortical cells - warrants a systematic investigation of the behavior of $2 \mathrm{D}$ systems. To address this question we study a network of mathematically simple phase oscillators. While the dynamics of pairs of oscillators $[15,16]$ or systems with simple connectivity schemes [17] are well understood, this is not the case for large networks with nontrivial connection schemes. Of general interest is the phase coupling that results in networks of oscillators with different coupling schemes.

In Sec. II we present the mathematical formulation of the phase-oscillator model and the three connection schemes studied in the subsequent sections of the paper. We studied three different $2 \mathrm{D}$ connection architectures: the simplest nearest-neighbor scheme, "Gaussian" schemes where each cell communicates with all its neighbors with the weights decreasing in a Gaussian manner up to a fixed cutoff, and a "sparse" connection pattern with a few connections per oscillator. The latter two geometries capture elements of cortical neuroanatomy which include locally dense connections and sparse longrange connections ranging over more than $6 \mathrm{~mm}$ [19].

Section III is devoted to numerical solutions of this system. In Sec. IV we derive analytic expressions for the correlation functions expected to arise from a linearized version of the interaction. In Sec. $V$ we develop an analytically tractable model for the sparse-connection scheme. Mathematical details will be delegated to the Appendix.

\section{MODEI}

The basic unit in our 2D networks is an oscillator whose phase $\theta_{i j}$ is $2 \pi$ periodic and which has the intrinsic frequency $\omega_{i j}$. The dynamics of an isolated oscillator are described by

$$
\frac{d \theta_{i j}}{d t}=\omega_{i j}
$$

The influence of the network can be expressed as an additional interaction term

$$
\frac{d \theta_{i j}}{d t}=\omega_{i j}+f_{i j}\left(\left\{\theta_{k l}\right\}\right) \text {. }
$$

In our model, the coupling function $f_{i j}$ is expressed as the sum of terms, each one consisting of the product of a global coupling strength $\alpha$, a connection-specific strength $J_{i j, k l}$, and the sine of a phase difference

$$
\frac{d \theta_{i j}}{d t}=\omega_{i j}-\alpha \sum_{k, l} J_{i j, k l} \sin \left(\theta_{i j}-\theta_{k l}\right) \text {. }
$$

This system, and numerous variants, has received a considerable amount of attention from solid-state physicists and applied mathematicians (see, e.g., $[12,15,18,17,21,20,22])$. However, most of these studies either only consider nearest-neighbor connectivities or they use a mean-field approximation. In this paper, we are concerned with connection architectures more relevant to cortical anatomy. We confine ourselves to three generic network configurations, all defined on a two-dimensional square lattice. These are characterized by the values of $J_{i j, k l}$ and the range over which the $(k, l)$ sum is taken, as described below.

(i) Nearest-neighbor connections: Fig. 1(a). In this often-used connection scheme, which we use as a reference for comparison with the other schemes described 
Nearest Neighbor

00000000

$\begin{array}{llllllllllllll}0 & 0 & 0 & 0 & 0 & 0 & 0 & 0\end{array}$

$0000-0 \begin{array}{ccccc}0 & 0 & 0 & 0\end{array}$

$\begin{array}{lllllllll}0 & 0 & 0 & 0 & 0 & 0 & 0 & 0\end{array}$

000000000

$\begin{array}{llllllllllllllllll}0 & 0 & 0 & 0 & 0 & 0 & 0 & 0\end{array}$
Gaussian 000000000 - 0 o g 9 g 0000 - 0 a a - 0 a- 0 - 0 o $00 / \phi$ br 0000000000 0000000000

\begin{tabular}{lllllllll}
\multicolumn{8}{c}{ Sparse } \\
0 & 0 & 0 & 0 & 0 & 0 & 0 & 0 & 0 \\
0 & 0 & 0 & 0 & 0 & 9 & 0 & 0 & 0 \\
0 & 0 & 0 & 0 & 0 & 0 & 0 & 0 & 0 \\
0 & 0 & 0 & 0 & & 0 & 0 & 0 & 0 \\
0 & 0 & 0 & 0 & 0 & 0 & 0 & 0 & 0 \\
0 & 0 & 0 & 0 & 0 & 0 & 0 & 0 & 0 \\
0 & 0 & 0 & 0 & 0 & 0 & 0 & 0 & 0
\end{tabular}

(c)
FIG. 1. Schematic representations of the three connection schemes discussed in the text. (a) The nearest-neighbor scheme, with four connections with equal weights per cell [Eq. (4)]; (b) the Gaussian-connection scheme [Eq. (5)] where each cell receives inputs from every neighbor within a specified radius and with weights that decay according to a Gaussian distribution; (c) the sparse-connection scheme [Eq. (6)]. In the sparse scheme each cell makes $n=5$ connections, each one being selected from a Gaussian distribution that makes the selection of short connections more likely. The sums of the weights of all connections are equal in all schemes.

below, each cell $(i j)$ is connected to each of its four neighbors:

$$
J_{i j, k l}\left\{\begin{array}{l}
=1 \text { if } k=i \pm 1 \text { and } l=j \pm 1 \\
=0 \text { otherwise } .
\end{array}\right.
$$

(ii) Gaussian connections: Fig. 1 (b). The cells are densely connected to every neighbor within a specified distance with Gaussian weighted connections. Hence

$$
J_{i j, k l}=\frac{1}{2 \pi \sigma} \exp \left[-\frac{(i-k)^{2}+(j-l)^{2}}{2 \sigma^{2}}\right] .
$$

We truncate this function at $2 \sigma$, i.e., $J_{i j, k l}=0$ if $|i-k| \geq 2 \sigma$ or $|j-l| \geq 2 \sigma$. While the connectivity in the nearest-neighbor case is $4, \sigma=2$ yields 27 connections per cell, and the largest network we studied, $\sigma=6$, results in 372 connections per cell.

(iii) Sparse connections: Fig. 1(c). In this scheme we no longer require symmetric connections, or that the connection pattern be deterministic from unit to unit. A given cell is connected to a fixed number $n$ of neighboring cells, with the probability of the connection from oscillator $(k, l)$ to oscillator $(i, j)$ determined by

$$
P_{i j, k l}=\frac{1}{2 \pi \sigma} \exp \left[-\frac{(i-k)^{2}+(j-l)^{2}}{2 \sigma^{2}}\right] .
$$

$J_{i j, k l}$ is unity with probability $P_{i j, k l}$ and zero otherwise. This connection scheme is generated for the lattice site $(i, j)$ by drawing $n$ coordinate pairs $(k, l)$ from a Gaussian distribution centered at $(i, j)$ that determines the indices of the cells that act upon the oscillator at location $(i, j)$, i.e., determines which indices appear in the sum of the right-hand side of Eq. (3) for index $(i, j)$. Therefore the probability of making a connection decreases with distance. The weight of all connections is the same for all connections and does not depend on the distance. We typically used $n=5$, and in all cases $2 \leq n \leq 10$.

The sum of the weights of all connections with a given oscillator $(i, j)$ was chosen identically in all three models, enabling us to study the efficacy of the different connection schemes for achieving phase locking. In contrast to most of the earlier studies, we do not restrict ourselves to the case of weakly interacting oscillators, but treat interaction-dominated systems, where $\alpha \sum_{k, l} J_{i j, k l} \gg \omega_{i j}$.

\section{CORRELATION FUNCTIONS FOR SPARSE AND GAUSSIAN CONNECTIVITIES}

We solved the system, Eq. (3), for a $128 \times 128$ system of oscillators, coupled according to one of the connection schemes specified by Eqs. (4)-(6) and using periodic boundary conditions (see below for initial conditions). We computed the time dependence of the usual two-point correlation function of phase coupling, defined as

$$
C(r, t)=\left\langle\cos \left[\theta_{i j}(t)-\theta_{k l}(t)\right]\right\rangle,
$$

where $r$ is defined as the separation between a pair of cells, $r=\left[(i-k)^{2}+(j-l)^{2}\right]^{1 / 2}$ and $-1 \leq C \leq 1$. Angular brackets denote the ensemble average over 10000 randomly selected pairs of oscillators. The intrinsic frequencies $\omega_{i j}$ are chosen randomly, with a Gaussian distribution with mean 0.5 and variance 1 . At $t=0$, phases are distributed randomly between 0 and $2 \pi$ with a uniform distribution (we have studied the influence of the initial conditions earlier [11]). In Fig. 2 we plot $C(r, t)$ for $r=20,30,40,50,60$, and 70. In these and the following figures, time is always plotted in units of the inverse of the average intrinsic oscillation frequency. The cases of Gaussian connectivity with $\sigma=6$ and hence $n=372$ connections per cell and of sparse connectivity with $\sigma=6$ and $n=5$ are presented in Figs. 2(a) and 2(b), respectively. The most striking difference is that correlation levels of over 0.9 are rapidly achieved in the sparse scheme for all cases, even for separations of 70 oscillators, while there are clear separation-dependent differences in the phase-locking behavior of the Gaussian model. In fact, even after $t=10$ there is no significant locking over the longer distances of $r=50,60$, or 70 units. For local connectivity schemes, like Gaussian connectivity with $\sigma=2$ or nearest-neighbor connections, no long-range order evolves even at larger times (data not shown).

Strong synchronization was also observed in another sparsely connected system. Satoh [23] compared phase locking in systems of van der Pol oscillators, with either nearest-neighbor coupling or with completely randomly distributed connections, i.e., without any geometry. $\mathrm{He}$ found, in accordance with our results, that the latter system showed much stronger synchronization than the former.

\section{CORRELATION FUNCTIONS FOR A LINEARIZED MODEL: NEAREST-NEIGHBOR INTERACTIONS}

In this section we investigate the time dependence of correlations between two phase angles separated by a distance $r$ in a rigorously solvable model of coupled oscillators. Our model is obtained by linearizing the interaction term in Eq. (3) for the nearest-neighbor connection 
scheme, i.e., by replacing $\sin \left(\theta_{i j}-\theta_{k l}\right)$ with $\left(\theta_{i j}-\theta_{k l}\right)$, which is justified for small $\left|\theta_{i j}-\theta_{k l}\right|$. Adding Gaussian, uncorrelated noise fields $\xi(\mathbf{x}, t)$, we obtain the equation of motion

$$
\dot{\theta}(\mathbf{x}, t)=\Omega(\mathbf{x})-\nabla^{2} \theta(\mathbf{x}, t)+\xi(\mathbf{x}, t),
$$

where we have passed to continuous space variables $\mathbf{x}$ with $\nabla^{2}$ representing the Laplacian and $\Omega(\mathbf{x})$ the local frequency. We have absorbed the interaction constant $\alpha$ by rescaling the time $t$ and in addition rescaled the Gaussian random frequency $\Omega(\mathrm{x})$ and noise fields $\xi(\mathrm{x}, t)$ appropriately, i.e., $\alpha t \rightarrow t, \quad \Omega(\mathrm{x}) / \alpha \rightarrow \Omega(\mathrm{x}), \xi(\mathrm{x}, t) / \alpha$
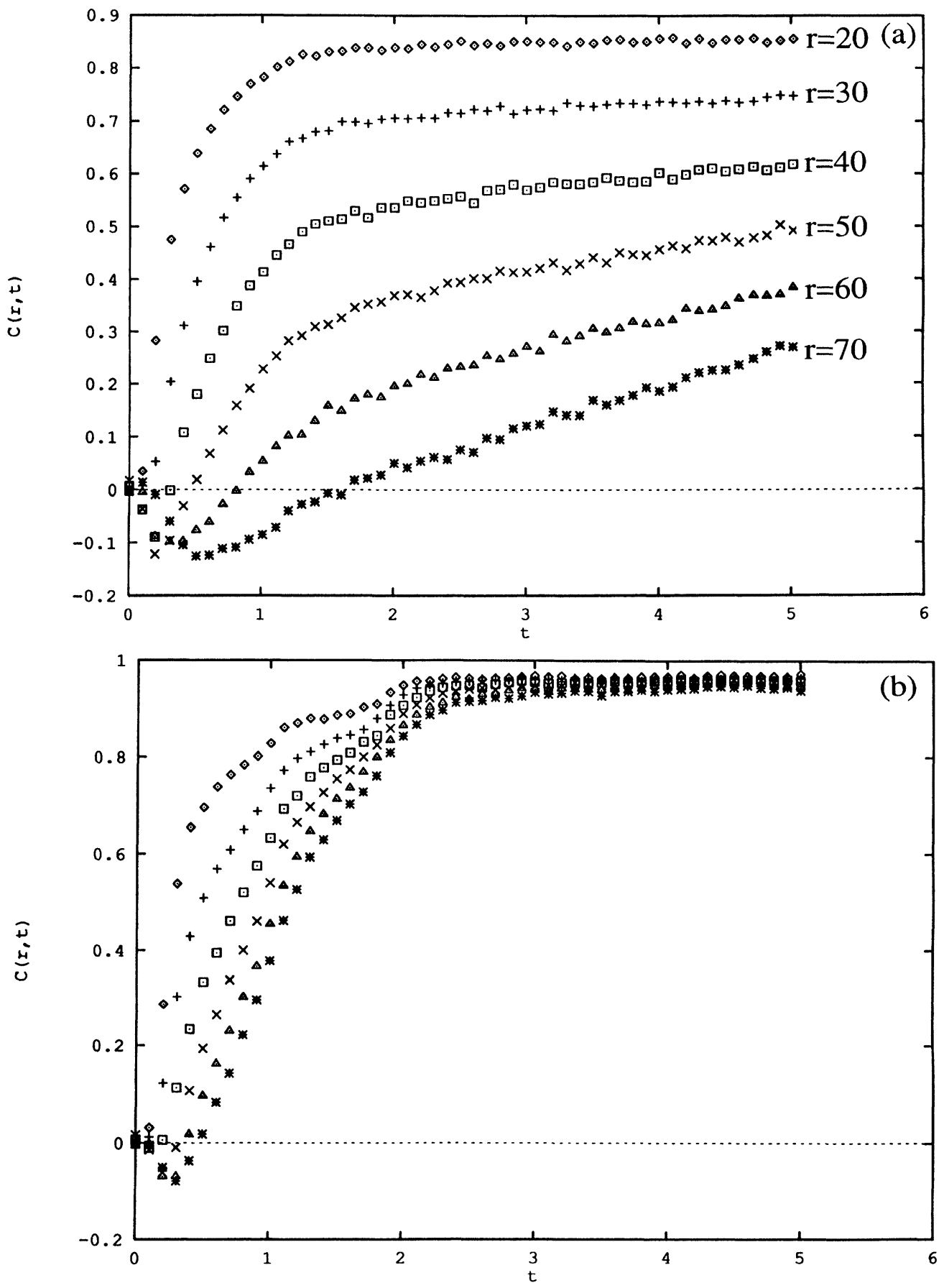

FIG. 2. Correlation functions for (a) the Gaussian and (b) the sparse-connection scheme $n=5$. In both cases, $\sigma=6$, $\alpha \sum_{k l} J_{i j, k l}=10$, and $N=128 \times 128$. Symbols (both plots): $r=20$, diamonds; $r=30$, plus signs; $r=40$, squares; $r=50$, crosses; $r=60$, triangles; and $r=70$, asterisks. In this and the following figures, time is measured in units of $\left(2 / N \sum_{i j} \omega_{i j}\right)^{-1}$, which is unity in this case. 
$\rightarrow \xi(\mathbf{x}, t)$. The variances of these random fields are specified by

$$
\begin{aligned}
& \left\langle\Omega(\mathbf{x}) \Omega\left(\mathbf{x}^{\prime}\right)\right\rangle_{\Omega}=\Delta^{2} \delta\left(\mathbf{x}-\mathbf{x}^{\prime}\right), \\
& \left\langle\xi(\mathbf{x}, t) \xi\left(\mathbf{x}^{\prime}, t^{\prime}\right)\right\rangle_{\xi}=2 T \delta\left(\mathbf{x}-\mathbf{x}^{\prime}\right) \delta\left(t-t^{\prime}\right),
\end{aligned}
$$

where $\Delta^{2}$ is the width of the distribution. Since Eq. (8) is linear in the angular variables it can be solved as

$$
\begin{gathered}
\theta(\mathbf{x}, t)=\int d \mathbf{x}^{\prime} \int d t^{\prime} G\left(\mathbf{x}-\mathbf{x}^{\prime}, t-t^{\prime}\right) \\
\times\left[\theta\left(\mathbf{x}^{\prime}, t^{\prime}\right) \delta\left(t^{\prime}\right)+\Omega\left(\mathbf{x}^{\prime}\right) H\left(t^{\prime}\right)\right. \\
\left.+\xi\left(\mathbf{x}^{\prime}, t^{\prime}\right) H\left(t^{\prime}\right)\right],
\end{gathered}
$$

where $H(t)$ is the Heaviside step function and $G\left(\mathbf{x}-\mathbf{x}^{\prime}, t-t^{\prime}\right)$ is the Green's function of Eq. (8) which obeys

$$
\left[\frac{\partial}{\partial t}+\nabla^{2}\right] G\left(\mathbf{x}-\mathbf{x}^{\prime}, t-t^{\prime}\right)=\delta\left(\mathbf{x}-\mathbf{x}^{\prime}\right) \delta\left(t-t^{\prime}\right) .
$$

The initial conditions in Eq. (11) were chosen as follows.

(i) At $t=0$, the angles $\theta\left(\mathbf{x}^{\prime}, 0\right)$ are distributed randomly with a Gaussian probability distribution with variance

$$
\left\langle\theta(\mathbf{x}, 0) \theta\left(\mathbf{x}^{\prime}, 0\right)\right\rangle=\kappa^{2} \delta\left(\mathbf{x}-\mathbf{x}^{\prime}\right),
$$

where $\kappa^{2}$ is the width of the distribution.

This leads to the term $\theta\left(\mathbf{x}^{\prime}, t^{\prime}\right) \delta\left(t^{\prime}\right)$ in Eq. (11).

(ii) The random frequencies $\Omega(x)$ of the oscillators and the time-dependent random fields $\xi(x)$ are switched on at time $t=0$. This generates the terms $\Omega\left(\mathbf{x}^{\prime}\right) H\left(t^{\prime}\right)$ and $\xi\left(\mathbf{x}^{\prime}, t^{\prime}\right) H\left(t^{\prime}\right)$, respectively, in Eq. (11).

Next we use the solution for $\theta(\mathrm{x}, t)$ given by Eq. (11) to compute the time-dependent correlation function

$$
C(\mathbf{x}, t)=\langle\exp \{i[\theta(\mathbf{x}, t)-\theta(0, t)]\}\rangle,
$$

where \langle\rangle denotes the average over the Gaussian fields $\theta(\mathbf{x}, 0), \Omega(\mathbf{x})$, and $\xi(\mathbf{x}, t)$. As could be expected from the additive character of the solution [Eq. (11)] and the independence of the random fields, $C(\mathbf{x}, t)$ factorizes as

$$
C(\mathbf{x}, t)=C_{\theta}(\mathbf{x}, t) C_{\xi}(\mathbf{x}, t) C_{\Omega}(\mathbf{x}, t),
$$

where $C_{\theta}(\mathbf{x}, t)$ is obtained by performing in Eq. (14) the average over the random initial conditions $\theta(\mathbf{x}, 0)$, etc. The individual factors for two dimensions $(d=2)$ and $t \geq 0$ are evaluated in the Appendix as

$$
\begin{gathered}
C_{\theta}(r, t)=\exp \left\{-\frac{\kappa^{2}}{2 t+1}\left[1-\exp \left[-\frac{r^{2}}{4(2 t+1)}\right]\right)\right\}, \\
C_{\xi}(r, t)=\exp \left[-2 T \int_{r^{2} / 4(2 t+1)}^{r^{2} / 4} d z \frac{1}{z}\left(1-e^{-z)}\right],\right. \\
C_{\Omega}(r, t)=\exp \left[-\Delta^{2} \frac{r^{2}}{4}\left[\int_{r^{2} / 4(t+1)}^{r^{2} / 4} d z f(z)\right.\right. \\
\left.\left.-\int_{r^{2} / 4(2 t+1)}^{r^{2} / 4(t+1)} d z f(z)\right]\right],
\end{gathered}
$$

where $r=|\mathbf{x}|$ and $f(z)=\left(1 / z^{2}\right) \int_{0}^{z}(1 / x)\left(1-e^{-x}\right) d x$. In the limit of short distances $r \ll<1$, the time behavior of the three different factors that comprise the correlation becomes transparent:

$$
\begin{aligned}
& C_{\theta}(r, t)=\exp \left[-\kappa^{2}\left(\frac{1}{2 t+1}\right)^{2} \frac{r^{2}}{4}\right], \\
& C_{\xi}(r, t)=\exp \left[-2 T\left(1-\frac{1}{2 t+1}\right) \frac{r^{2}}{4}\right], \\
& C_{\Omega}(r, t)=\left[\frac{1+2 t}{(1+t)^{2}}\right]^{\Delta^{2} r^{2} / 4} .
\end{aligned}
$$

These curves are plotted separately in Fig. 3(a), and are combined to yield the full correlation function [Eq. (15)] in Fig. 3(b). The most striking aspect of the model is the slow decay of all correlations due to the power law in the $C_{\Omega}(r, t)$ term. The interpretation of the evolution of these components of the correlation function in time is as follows.

(i) If the random fields $\Omega(x)$ and $\xi(x, t)$ are switched off, the space-time dependence of correlations is described by $C_{\theta}(r, t)$ [top curve, Fig. 2(a)]. The system is in this situation purely diffusive. Random initial angles separated by a distance $r$ will slowly align by diffusion, i.e., their correlation function tends to 1 for $t \rightarrow \infty$.

(ii) The correlation function $C_{\xi}(r, t)$ describes the evolution of angular correlations in the case where all angles have at time $t=0$ the same value $\theta(\mathbf{x}, 0)=\theta$ and all random frequencies are zero, $\Omega(x)=0$. Under the influence of the time-dependent random field $\xi(\mathbf{x}, t)$ the differences between different angles start to grow. In the long-time limit the correlation function approaches the thermal equilibrium value $C_{\xi}(r, t \rightarrow \infty)$, which means that phase angles at different sites remain correlated but that the correlations decay exponentially with distance.

(iii) $C_{\Omega}(r, t)$ describes how in the absence of timedependent disturbances $[\xi(\mathbf{x}, t)=0]$ initially equal angles $[\theta(\mathbf{x}, 0)=\theta$ for all $\mathbf{x}]$ "align" themselves locally to the angles determined by the static random frequencies $\Omega(\mathbf{x})$. The interaction term cannot counterbalance this effect and correlations at all distances decay to zero for large time. Long-time synchronization cannot therefore be established in a two-dimensional system with only local interactions when the oscillators have a random distribution of intrinsic frequencies. This result has been shown earlier by Sakaguchi, Shinomoto, and Kuramoto [15], who showed that assumption of the contrary leads to a contradiction. In the following, the absence of long-time synchronization will be confirmed numerically.

Figures 4(a) and 4(b) present a numerical comparison between $C(r, t)$ of the linear model from Eq. (8) and the nonlinear model with nearest-neighbor interactions. We see that for the linear model the phases rapidly phase lock because the interaction potential, which has the form of a cosine for the nonlinear model, is replaced in the linearized version by a parabola which has a strong phase-locking effect. For longer times, however, the phases drift farther apart in the linear model than in the nonlinear version because in the linear model the interac- 
tion is not periodic, i.e., there is no modulo function to restrict the absolute phase difference. Thus for $t \rightarrow \infty$ the power-law decay in the correlation function arising from the $C_{\Omega}(r, t)$ term [Eq. (19)] dominates the interaction. The combination of these two effects - strong phase lock- ing at early times, and drifting apart of the phases even of nearest neighbors at later times-leads to the nonmonotonic behavior of the correlation function that is observed for all connectivities in the linearized model.

Solutions of the full nonlinear system [Eq. (3), with
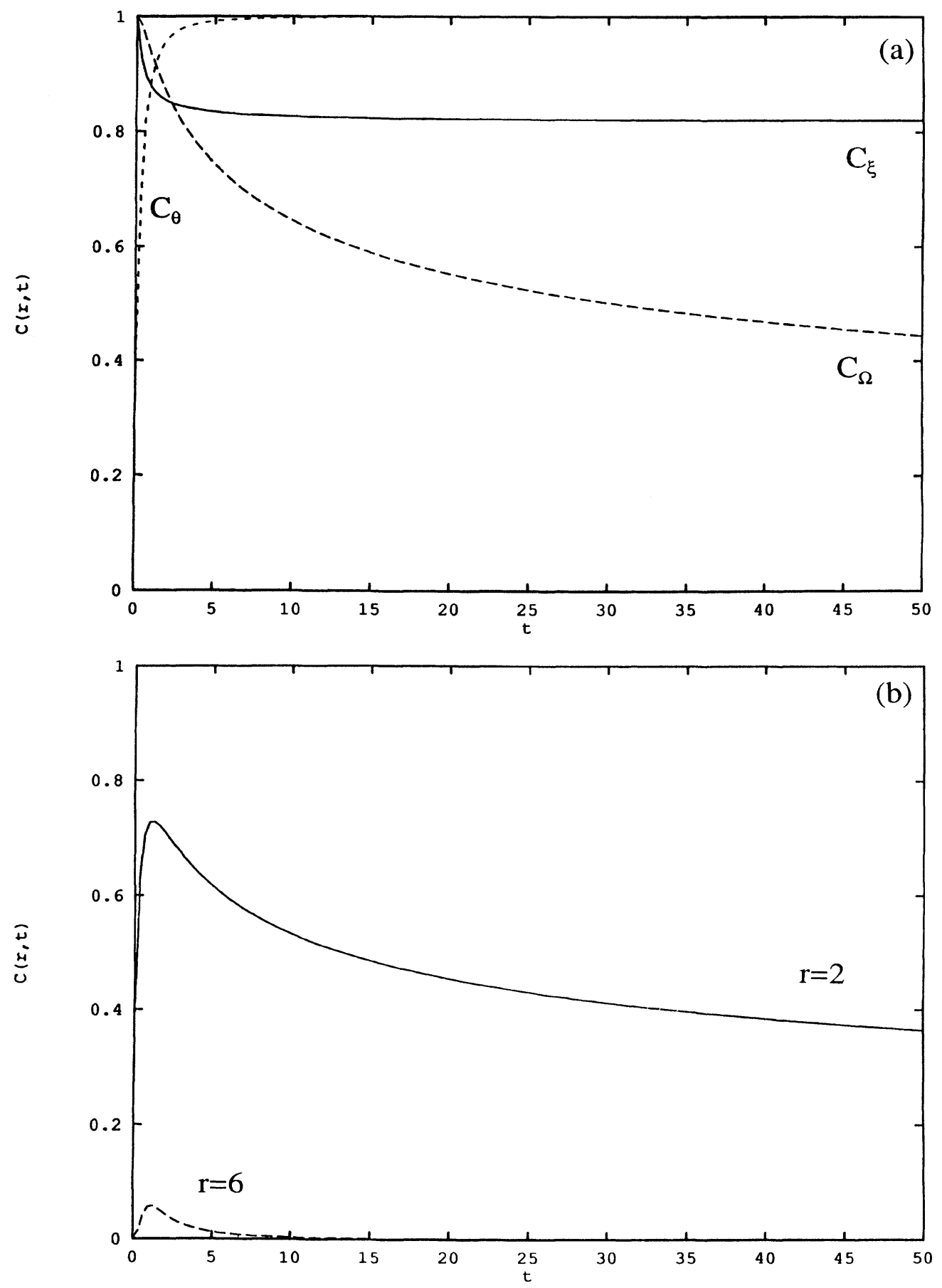

FIG. 3. (a) The $C_{\xi}(\mathbf{x}, t)$ (solid line), $C_{\theta}(\mathbf{x}, t)$ (short-dashed line), and $C_{\Omega}(\mathbf{x}, t)$ (long-dashed line) separable components of the linearized correlation function for nearest-neighbor connectivity [Eqs. (19) $-(21)$ ]. Parameters: $\Delta=0.5, T=0.1, \kappa=1, r=2$. (b) The product correlation function for the linearized nearest-neighbor coupled system, $C_{\theta}(\mathbf{x}, t) C_{\xi}(\mathbf{x}, t) C_{\Omega}(\mathbf{x}, t)$, Eq. (15). The curves are for separations of $r=2$ (solid line) and $r=6$ (dashed line), respectively. Other parameters are the same as in (a). 

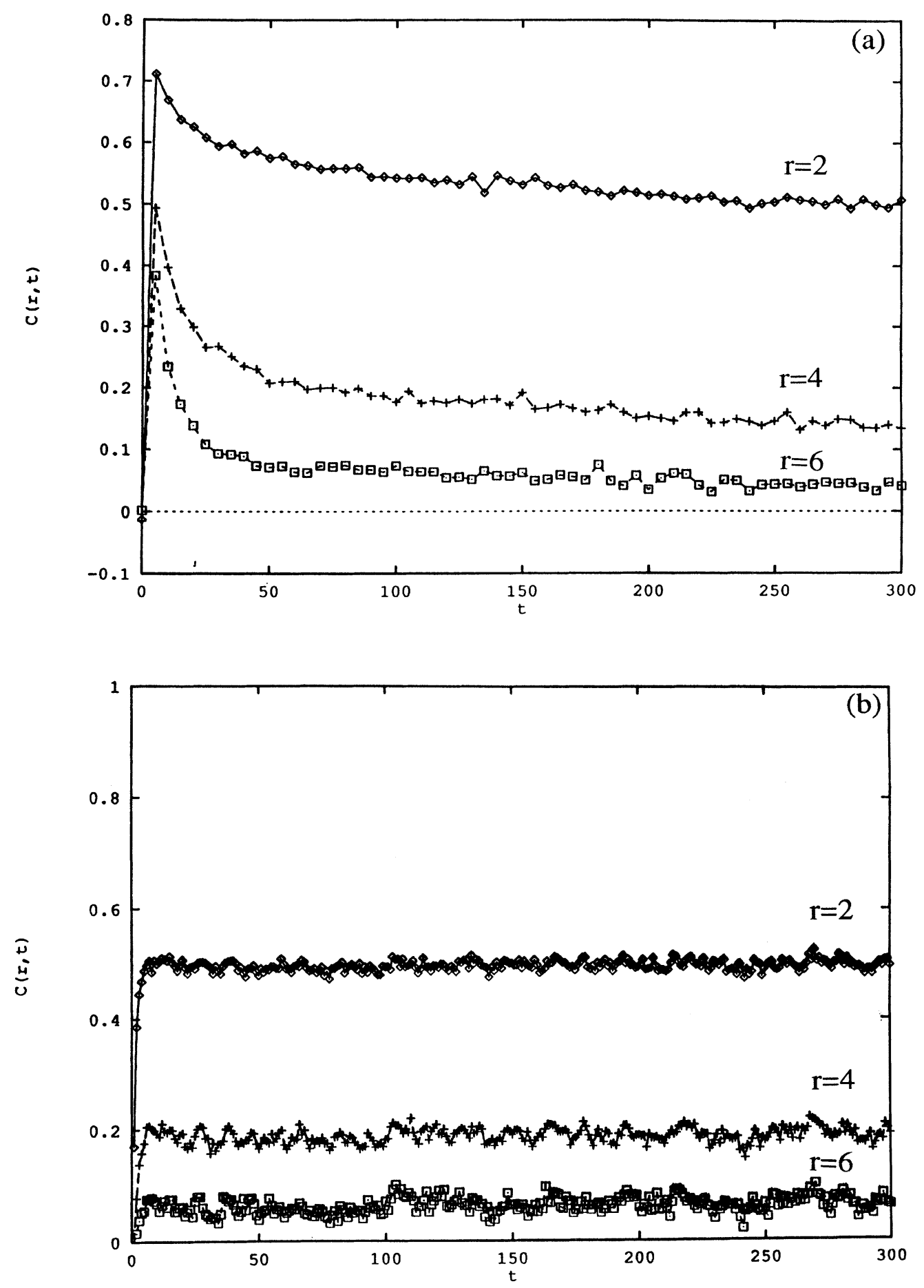

FIG. 4. (a) Correlation functions for the $N=128 \times 128$ oscillator system in the linearized model of Eq. (8), using nearest-neighbor connectivity. The spatial separations range from $r=2$ (diamonds) to $r=4$ (plus signs) to $r=6$ (squares). Parameters: $\alpha \sum_{k l} J_{i j, k l}=1$, $T=0$. Intrinsic frequencies are distributed uniformly between 0 and 1 , phases at $t=0$ are distributed uniformly between 0 and $2 \pi$. For separations above $r=6$ the correlation is virtually zero at all times. Note the decay in correlation over time, as predicted by the correlation model plotted in Fig. 3(b). (b) Correlation functions for the $N=128 \times 128$ oscillator system in the nonlinear model of Eq. (3), using nearest-neighbor connectivity. Parameters: $\Delta=0.5$; others are the same as in (a). The spatial separations range from $r=2$ (diamonds) to $r=4$ (plus signs) to $r=6$ (squares). The $2 \pi$ periodicity of the interaction term maintains all phase differences within a small range, resulting in the flat correlation function. (c) Ensemble correlation function of Eq. (23), plotted for $r=2$ (solid line), $r=6$ (long-dashed line), and $r=20$ (short-dashed line), for $\gamma=0.1$. Other parameters are the same as in Fig. 3(a). Note the long-range correlation, which is absent in Fig. 3(b). 


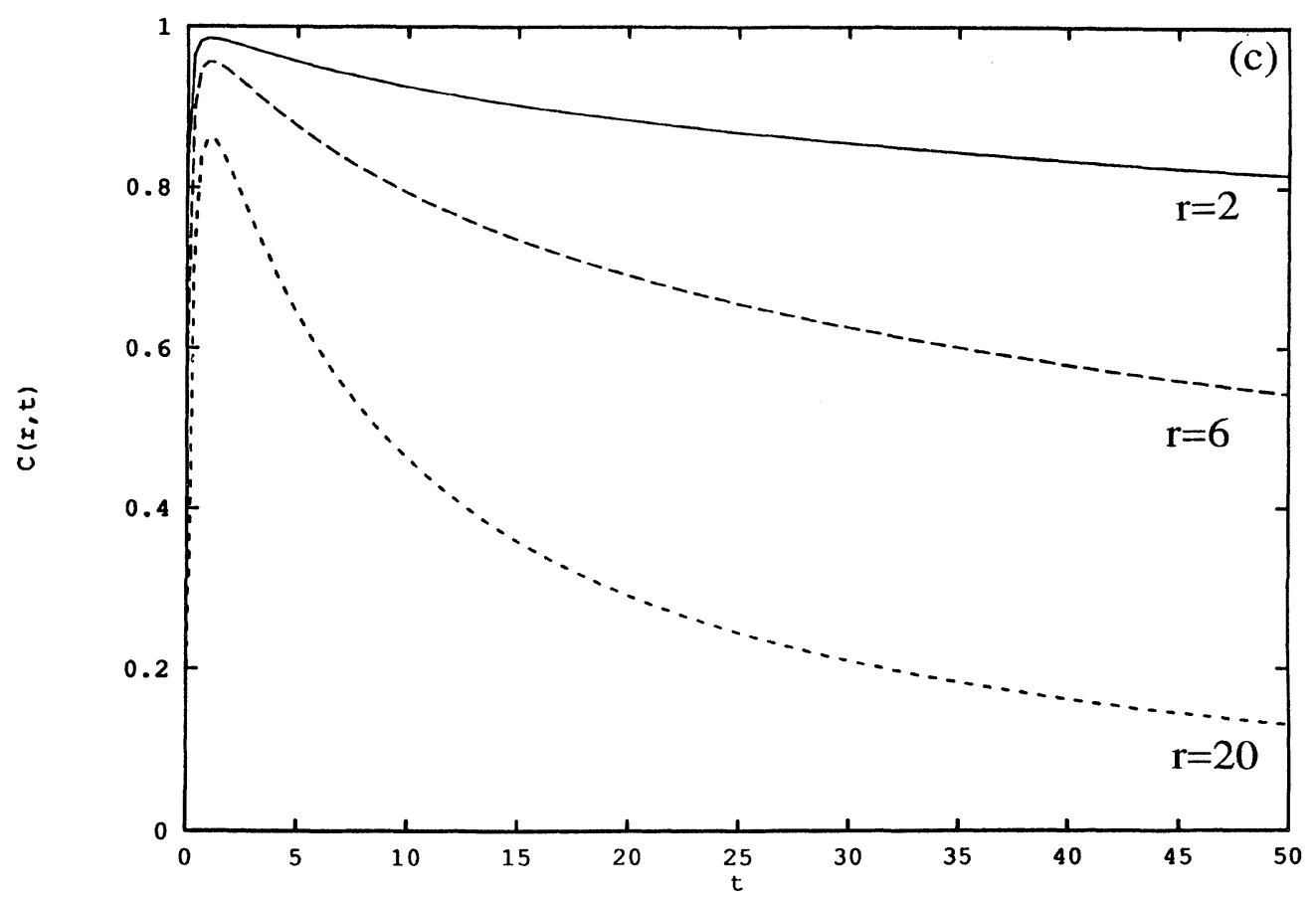

FIG. 4. (Continued).

plots in Fig. 4(b)] exhibit somewhat different behavior, particularly in the long-time limit where the presence of the $2 \pi$ periodicity becomes dominant in the nonlinear model. With phase differences constrained to lie in a finite range, the correlation between spatially separated oscillators remains constant in the full model, deviating from the behavior of the linear model due to the presence of a periodic interaction as mentioned above.

\section{A MODEL FOR SPARSE LONG-RANGE CONNECTIVITY}

The lack of long-range order is an important constraint on connection schemes that may subserve global phase coherence. It thus becomes important to understand the role of long-range-sparse-interaction schemes which are both motivated biologically and, as shown by numerical experiments in Sec. III of this article, are able to generate rapid phase synchronization. Even in a linear model the problem of percolation with interactions of different ranges cannot be solved rigorously. We therefore resort to a simpler model to understand the principle by which sparse long-range interactions speed up synchronization. We explore a nearest-neighbor model, which on a square lattice yields four connections of equal weight per cell.
For fixed lattice constant $a$, this system is essentially equivalent to the one investigated in Sec. IV. The only new element is that distances have to be measured in units of the lattice constant $a$, i.e., we have to replace $r$ in all correlation functions by $r / a$.

Consider an ensemble of these systems with different lattice constants $a$ and associate with each member of the ensemble a probability of connection $\exp \left(-\gamma a^{2}\right)$, i.e., long-range interactions with larger $a$ values have the same strength as short-range interactions, but occur with exponentially smaller probabilities. In this way we mimic the effect of long-range sparse interactions. Averaging the correlation function over the ensemble thus reduces to a Gaussian integral over the correlation functions, i.e.,

$$
\langle C(r, t)\rangle_{a}=\int_{0}^{\infty} d a e^{-\gamma a^{2}} C(r / a, t) / \int_{0}^{\infty} d a e^{-\gamma a^{2}} .
$$

It is shown in the Appendix that the effect of this average is to replace in the product $C(r, t)$ of the correlation functions given by Eqs. (19) $-(21)$ the exponent by its square root times a factor $\gamma$, which characterizes the width of the distribution of interaction ranges $a$. Integrating Eq. (22) then leads to

$$
\langle C(r, t)\rangle_{a}=\exp \left\{-\gamma r\left[\left(\frac{\kappa}{2 t+1}\right]^{2}+2 T\left[1-\frac{1}{2 t+1}\right]^{2}+\Delta^{2} \ln \left[\frac{(1+t)^{2}}{1+2 t}\right]\right]^{1 / 2}\right\} .
$$


Correlation functions for this connection scheme, shown in Fig. 4(c) for $r=2$ (solid line), $r=6$ (long-dashed line), and $r=20$ (short-dashed line), demonstrate that the presence of long-range interactions which occur with exponentially small probabilities leads to a higher level of synchronization, as is found numerically. Eventually, however, the correlations will decay due to the last term in Eq. (23).

\section{CONCLUSIONS}

It has been shown that for a linear model of coupled oscillators, Eq. (8), the time evolution of phase correlations is influenced differently by the interaction between the phases, by the intrinsic frequencies, and by timedependent random noise (which may simulate the effect of otherwise-neglected degrees of freedom in the system). The phase correlation function separates into three factors with different time dependencies. The buildup of correlations, i.e., the synchronization effect, is due to the deterministic interaction term. The time-dependent noise tends to destroy correlations, but the interaction term is still strong enough to provide finite correlations between angles separated by finite distances. The static random frequencies are in two dimensions strong enough to overcome the interaction term. Although there are finite correlations for short times, they decay to zero in the infinite time limit as the angular motion tends to follow the random local frequencies. Finally, it has been shown that finite long-range interactions which occur with exponentially small probabilities for long distances lead to fast synchronization over short time scales, as has been found numerically.

Numerical solutions of the coupled-oscillator model, Eq. (2), show that sparse long-range interactions lead to a more rapid and robust phase locking of oscillators than short-range Gaussian connections with the same overall coupling strength. In many ways, Gaussian and sparse connection schemes represent opposing avenues to achieve global phase coherence: exhaustive local coupling or distributed long-range coupling. Long-range sparse connections could be neurophysiologically realized in the visual cortex by the small fraction of axons that are observed to span distances over $6 \mathrm{~mm}$. This might explain the recent experiments $[7,8]$ where coherent oscillations between different areas of the visual cortex and even across the two hemispheres in cat brains have been observed. From the theoretical point of view, the understanding of the effect of long-range sparse connections that are randomly distributed on a lattice on the phase locking of nonlinear oscillators remains an interesting problem to be solved. It is closely related to percolation on a lattice with long-range interactions. A solution of this problem could have an important impact on our understanding of realistic models of neural networks and related systems.

\section{ACKNOWLEDGMENTS}

We would like to thank D. Ruderman for developing and implementing part of the Connection Machine code, and $\mathbf{H}$. Sompolinsky for stimulating discussions. Parts of the computations were performed on the CaltechArgonne Connection Machine. E.N. is supported by the Swiss National Science Foundation through Grant No. 8220-25941. H.G.S. is supported by the Volkswagen Foundation. D.M.K. received support from the Weizmann foundation. C. K. acknowledges support from the U. S. Air Force Office of Sponsored Research, from the NSF, and from the James S. McDonnell Foundation.

\section{APPENDIX: COMPUTATION OF THE CORRELATION FUNCTIONS}

In order to compute the different factors $C_{\theta}(r, t)$, $C_{\xi}(r, t)$, and $C_{\Omega}(r, t)$ appearing in Eq. (15), we first solve Eq. (12) by Fourier transformation and obtain in $d$ dimensions

$G(\mathbf{x}, t)=\int d^{d} q \int d \omega e^{-i \omega t} e^{-i \mathbf{q} \cdot \mathbf{x}}\left(-i \omega+q^{2}\right)^{-1}$.

This yields for Eq. (11)

$$
\begin{aligned}
\theta(\mathbf{x}, t)=\int d^{d} q \int d \omega e^{-i \mathbf{q} \cdot \mathbf{x}} e^{-i \omega t}\left(-i \omega+q^{2}\right)^{-1} \\
\times(\theta(\mathbf{q}, 0)+H(\omega) \Omega(\mathbf{q}) \\
\left.\quad+\int d \omega^{\prime} H\left(\omega-\omega^{\prime}\right) \xi\left(\mathbf{q}, \omega^{\prime}\right)\right)
\end{aligned}
$$

where $H(\omega)=\lim _{\epsilon \rightarrow 0^{+}}(\epsilon-i \omega)^{-1}$ is the Fourier transform of the Heaviside function and $\theta(\mathbf{q}, 0), \Omega(\mathbf{q})$, and $\xi(\mathbf{q}, \omega)$ are the Fourier transforms of the corresponding spaceand time-dependent quantities.

By using the following three properties:

(i)

$$
\begin{aligned}
\left\langle e^{i 2 x a}\right\rangle & =\int d x e^{-x^{2} / \kappa^{2}} e^{2 i x a} / \int d x e^{-x^{2} / \kappa^{2}} \\
& =e^{-a^{2} \kappa^{2}}=\exp \left(-a^{2}\left\langle x^{2}\right\rangle\right)
\end{aligned}
$$

(ii) $\left\langle\xi(q, \omega) \xi\left(q^{\prime}, \omega^{\prime}\right)\right\rangle=2 T \delta\left(\omega+\omega^{\prime}\right) \delta\left(\mathbf{q}+\mathbf{q}^{\prime}\right)$;

(iii) the independence of the averages over the different variables $\theta(\mathbf{x}, 0), \Omega(\mathbf{x})$, and $\xi(\mathbf{x}, t)$; we obtain for a $2 D$ system

$$
\begin{aligned}
& C_{\theta}(r, t)=\exp \left(-\kappa^{2} I_{\theta}\right), \\
& \begin{aligned}
I_{\theta} & =\int d^{2} q\left|\int d \omega e^{-i \omega t}\left(-i \omega+q^{2}\right)^{-1}\right|^{2}\left(1-e^{-i q \cdot \mathbf{x}}\right) \\
& =\int d^{2} q e^{-2 q^{2} t}\left(1-e^{-i \mathbf{q} \cdot \mathbf{x}}\right) \\
& =\int d^{2} q e^{-q^{2}-2 q^{2} t}\left(1-e^{-i \mathbf{q} \cdot \mathbf{x}}\right) \\
& =\frac{1}{2 t+1}\left[1-\exp \left[\frac{-r^{2}}{4(2 t+1)}\right]\right] .
\end{aligned}
\end{aligned}
$$

Note that we have introduced a cutoff in $q$ space via a factor $e^{-q^{2}}$ because in discrete systems all $q$ values are bounded from above by $2 \pi / l$ where $l$ is the lattice constant. (Here all distances are measured in units of $l$, i.e., $l=1$.)

By proceeding along the same lines as above, $C_{\xi}(r, t)$ becomes 


$$
\begin{aligned}
& C_{\xi}(r, t)=\exp \left(-\Delta^{2} I_{\xi}\right), \\
& \begin{aligned}
I_{\xi} & =\lim _{\epsilon \rightarrow 0} \int d^{2} q \int d \omega\left|\int d \omega^{\prime} e^{-i \omega t}\left(-i \omega^{\prime}+q^{2}\right)^{-1}\left(-i \omega-i \omega^{\prime}+\epsilon\right)^{-1}\right|^{2}\left(1-e^{-i \mathbf{q} \cdot \mathbf{x}}\right) \\
& =\int d^{2} q \frac{1}{q^{2}}\left(1-e^{-2 q^{2} t}\right)\left(1-e^{-i \mathbf{q} \cdot \mathbf{x}}\right) \\
& =\int d^{2} q e^{-q^{2}}\left(1-e^{-2 q^{2} t}\right)\left(1-e^{-i \mathbf{q} \cdot \mathbf{x}}\right) / q^{2} \\
& =\int_{0}^{\infty} d s \int d^{2} q e^{-(s+1) q^{2}\left(1-e^{-2 q^{2} t}\right)\left(1-e^{-i \mathbf{q} \cdot \mathbf{x}}\right)=\int_{r^{2} / 4(2 t+1)}^{r^{2} / 4} d z \frac{1}{z}\left(1-e^{-z}\right) .}
\end{aligned}
\end{aligned}
$$

Finally, we obtain for $C_{\Omega}(r, t)$

$$
\begin{aligned}
& C_{\Omega}(r, t)=\exp \left(-\Delta^{2} I_{\Omega}\right) \\
& I_{\Omega}=\int d^{2} q\left|\int d \omega e^{-i \omega t}(-i \omega+\epsilon)^{-1}\right|^{2}\left(1-e^{-i \mathbf{q} \cdot \mathbf{x}}\right) \\
& \quad=\int d^{2} q\left(1-e^{-q^{2} t}\right)^{2}\left(1-e^{-i \mathbf{q} \cdot \mathbf{x}}\right) / q^{4} \\
& \quad=\int d^{2} q \int_{0}^{\infty} d v e^{-v q^{2}} \int_{0}^{\infty} d s e^{-s q^{2}} e^{-q^{2}\left(1-e^{-q^{2} t}\right)^{2}\left(1-e^{-i \mathbf{q x}}\right) \frac{r^{2}}{4}\left[\int_{r^{2} / 4(t+1)}^{r^{2} / 4} d z f(z)-\int_{r^{2} / 4(2 t+1)}^{r^{2} / 4(t+1)} d z f(z)\right)}
\end{aligned}
$$

where $f(z)=\left(1 / z^{2}\right) \int_{0}^{z} \frac{1}{x}\left(1-e^{-x}\right) d x$.

The averages over the interaction range $a$ in Eq. (22) involve [if one takes into account the special form of Eqs. (19)-(21)] the following integral:

$$
I=\int_{0}^{\infty} d a e^{-\gamma^{2} a^{2}} e^{-A / a^{2}} / \int_{0}^{\infty} d a e^{-\gamma^{2} a^{2}},
$$

where the factor $A$ is different in each of the three equations. Since

$$
e^{-A / a^{2}}=\sqrt{\pi} a \int_{-\infty}^{\infty} d y e^{-a^{2} y^{2}} e^{2 i y \sqrt{A}},
$$

the $a$ integration in Eq. (A11) can be performed, yielding

$$
I=\frac{\gamma}{\pi} \int_{-\infty}^{\infty} d y \frac{1}{y^{2}+\gamma^{2}} e^{2 i y \sqrt{A}}=e^{-2 \gamma \sqrt{A}} .
$$

*Address correspondence to E.N. at Division of Biology, 216-76, Caltech, Pasadena, CA 91125. Electronic address: ernst@cns.caltech.edu.

†Permanent address: Institut für Theoretische Physik, Universität Kiel, 2300 Kiel 1, Germany.

$\ddagger$ Present address: Department of Physics, Harvard University, Cambridge MA 02138. Electronic address: kammen@ hrl.havard.edu.

[1] D. R. Tilley, Phys. Lett. 33A, 205 (1970).

[2] S. S. Wang and H. G. Winful, Appl. Phys. Lett. 52, 1774 (1988).

[3] I. Rodriguez-Iturbe, D. Entekhabi, and R. L. Bras, MIT Center for Global Change Science Report No. 5, 1990 (unpublished).

[4] A. H. Cohen, P. J. Holmes, and R. H. Rand, J. Math. Biol. 13, 345 (1982); N. Kopell and G. B. Ermentrout, Math. Biosci. 90, 87 (1988); N. Kopell, in Neural Control of Rhythmic Movements in Vertebrates, edited by A. H. Cohen, S. Rossignol, and S. Grillner (Wiley, New York, 1988).

[5] W. J. Freeman, Elect. Clin. Neurophys. 44, 586 (1978).

[6] E. Pöppel and N. Logothetis, Naturwissenschaften 73, 267 (1986); C. Madler and E. Pöppel, ibid. 74, 42 (1987).

[7] R. Eckhorn, R. Bauer, W. Jordan, M. Brosch, W. Kruse,
M. Munk, and H. J. Reitboeck, Biol. Cybern. 60, 121 (1988).

[8] C. M. Gray and W. Singer, Proc. Natl. Acad. Sci. U.S.A. 86, 1698 (1989); C. M. Gray, P. König, A. K. Engel, and W. Singer, Nature 338, 334 (1989); A. K. Engel, P. König, A. K. Kreiter, and W. Singer, Science 252, 1177 (1991).

[9] C. von der Malsburg and W. Schneider, Biol. Cybern. 54, 29 (1986); F. Crick and C. Koch, Semin. Neurosci. 2, 263 (1990).

[10] D. M. Kammen, P. J. Holmes, and C. Koch, in Models of Brain Function, edited by R. M. J. Cotterill (Cambridge University Press, Cambridge, 1989); p. 273.

[11] E. Niebur, D. M. Kammen, and C. Koch, in Nonlinear Dynamics and Neuronal Networks, edited by W. Singer and H. G. Schuster (VCW Verlag, Weinheim, 1990), p. 173; E. Niebur, D. M. Kammen, C. Koch, D. Ruderman, and H. G. Schuster, in Advances in Neural Information Processing Systems 3, edited by D. S. Touretzky and R. Lippmann (Morgan-Kaufman, Palo Alto, 1991), p. 123.

[12] E. Niebur, H. G. Schuster, and D. M. Kammen, Phys. Rev. Lett. (to be published).

[13] M. A. Wilson and J. Bower, Neural Comput. (to be published).

[14] H. Sompolinsky, H. Golomb, and D. Kleinfeld, Proc. 
Natl. Acad. Sci. U.S.A. 87, 7200 (1990); H. Sompolinsky, H. Golomb, and D. Kleinfeld, Phys. Rev. A 436990 (1991).

[15] H. Sakaguchi, S. Shinomoto, and Y. Kuramoto, Prog. Theor. Phys. 77, 1005 (1987); 79, 1069 (1988).

[16] H. G. Schuster and P. Wagner, Biol. Cybern. 64, 77 (1990); 64, 83 (1990).

[17] S. H. Strogatz and R. E. Mirollo, J. Phys. A 21, L699 (1988); P. C. Mathews and S. H. Strogatz, Phys. Rev. Lett. 65, 1701 (1990).

[18] G. B. Ermentrout and N. Kopell, SIAM J. Appl. Math.
50, 125 (1990).

[19] C. D. Gilbert and T. N. Wiesel, J. Neurosci. 6, 1160 (1986); E. White, Cortical Circuits (Birkhäuser, Boston, 1989).

[20] R. Loft and T. A. DeGrand, Phys. Rev. B 35, 8528 (1987).

[21] Y. Kuramoto, Chemical Oscillations, Waves, and Turbulence (Springer-Verlag, Heidelberg, 1984).

[22] J. M. Kosterlitz and D. J. Thouless, J. Phys. C 6, 1181 (1973); J. M. Kosterlitz, ibid. 7, 1046 (1974).

[23] K. Satoh, J. Phys. Soc. Jpn. 58, 2010 (1989). 